\title{
Comparative Study of Linear and Nonlinear Ultrasonic Techniques for Evaluation Thermal Damage of Tube-Like Structures
}

\author{
Weibin $\mathrm{Li}^{*}$, Younho Cho* ${ }^{\dagger}$ and Xianqiang $\mathrm{Li}^{*}$
}

\begin{abstract}
Ultrasonic guided wave techniques have been widely used for long range nondestructive detection in tube-like structures. The present paper investigates the ultrasonic linear and nonlinear parameters for evaluating the thermal damage in aluminum pipe. Specimens were subjected to thermal loading. Flexible polyvinylidene fluoride (PVDF) comb transducers were used to generate and receive the ultrasonic waves. The second harmonic wave generation technique was used to check the material nonlinearity change after different heat loadings. The conventional linear ultrasonic approach based on attenuation was also used to evaluate the thermal damages in specimens. The results show that the proposed experimental setup is viable to assess the thermal damage in an aluminum pipe. The ultrasonic nonlinear parameter is a promising candidate for the prediction of micro-damages in a tube-like structure.
\end{abstract}

Keywords: PVDF Transducer, Thermal Damage, Nonlinear Parameter, Attenuation

\section{Introduction}

Tube-like structures have been widely used in the oil, gas, and petro-chemical industry. In these industries, structures are usually subjected to thermal loading frequently. In metallic materials, the micro-structure gradually changes under the effect of the external environment [1]. Thermal loading is an in-service condition for structural components. The general defects caused by thermal loading include creep, corrosion, efflorescence, ect. Therefore, a nondestructive approach to accurately evaluate thermal damage in tube-like structures is essential.

The ultrasonic guided wave technique is one of the most widely used nondestructive evaluation (NDE) methods. It can propagate over considerable distances without point-by-point scanning and the wave propagation interacts throughout the entire thickness of the structure, which means that it can find both surface and internal defects. Low energy consumption and great cost-effectiveness are features of the guided wave-based damage detection approach [4,5] Most conventional guided NDE methods that the use of linear ultrasonic characteristics are primarily used to evaluate the properties of the material. The nonlinear ultrasonic technique based on the nonlinear ultrasonic behaviors such as nonlinear resonance, mixed frequency response, sub-harmonic generation, or higherharmonic generation, has been studied as a promising method to overcome the limitation $[2,3]$. However, according to the previous work, a number of investigators have applied nonlinear ultrasonic techniques to detect micro-damages in different metallic alloys of plate structure, while there have been only few works reported on nonlinear ultrasonic research on the tube-like structures.

The objective of this paper is to evaluate the thermal damage by linear and nonlinear ultrasonic techniques. Measurements of variation of attenuation, and normalized second harmonic amplitude wave carried out to check the damage status in the specimens. 


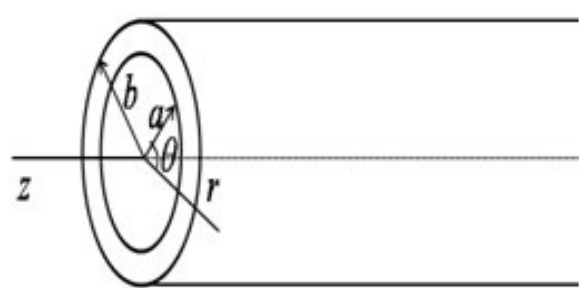

Fig. 1 Cylindrical shell

\section{Fundamental Theory}

This problem of circumferential wave propagation was studied by Viktorov (1967) and Qu, Berthelot, and Li (1996). Consider time-harmonic $\left(e^{-i w t}\right)$ waves propagating in the $\theta$ direction in the $(r, \theta)$ plane. The axial direction $\mathrm{z}$ is perpendicular to the cylinder cross-section and the elastic field does not depend on the coordinate $z$. The equation of motion can be obtained from the governing Navier wave equations in cylindrical coordinates, as show in Fig. 1.

\subsection{Linear Ultrasonic Test}

The present paper also considers linear ultrasonic wave techniques used in specimens under different thermal loadings to detect damage to compare the sensitivity of linear and nonlinear acoustic parameters to thermal damage in velocity of the same longitudinal mode.

The amplitude decay is assumed to be mainly affected by attenuation. The attenuation is an important characteristic of guided wave propagation [6,7]. It refers to the reduction in the signal strength after a wave travels some distance. The wave attenuation is defined by an attenuation coefficient. In this work, a conventional guided wave pulse-echo setup is used to measure the attenuation factor by comparing the two amplitudes of a given mode captured at the two corresponding traveling distances. The attenuation is denoted by $\alpha$. It uniquely defines the decrease of wave amplitude depending on frequency and mode as follows,

$$
\ln \frac{A x_{1}}{A x_{2}}=\alpha\left(x_{1}-x_{2}\right)
$$

where $A x_{1}$ and $A x_{2}$ are the amplitudes of a wave mode signal obtained at the two corresponding propagation distances $x_{1}$ and $x_{2}$ respectively.

In general, a guided wave propagates as a wave packet containing a number of neighboring frequency components around the center frequency; the speed of the wave packet is called the group velocity. It also defines the velocity of energy carried by the wave packet. In this paper, the experimental group velocity of a guided wave can be easily verified by comparing the time difference in an oscilloscope between the two different receiver positions over a give span with the theoretically predicted value, which is calculated via numerical differentiation from the phase velocity dispersion curve. The guided wave's group velocity essentially varies with the change of material properties. As a wave travels across a damaged area of reduced stiffness, the group velocity will be affected.

\subsection{Nonlinear Ultrasonic Test}

The method to detect nonlinearity is to launch an ultrasonic guided wave of a given frequency into the specimen, and receive the signal after a certain propagation distance. Due to the material nonlinearity, after propagation over a certain distance, the fundamental frequency wave will distort and generate higher harmonics [8-11].

For a guided wave, acoustic nonlinearity could be written analogously as

$$
\beta=\frac{8}{K^{2} a} \frac{A_{2}}{A_{1}^{2}} f,
$$


Table 1 Various temperatures of heat loading

\begin{tabular}{|c|c|c|c|c|}
\hline Material & Melting point $\left({ }^{\circ} \mathrm{C}\right)$ & Reply temperature $\left({ }^{\circ} \mathrm{C}\right)$ & $\begin{array}{c}\text { Recrystallization } \\
\text { temperature }\left({ }^{\circ} \mathrm{C}\right)\end{array}$ & $\begin{array}{c}\text { Recrystallization annealing } \\
\text { temperature }\left({ }^{\circ} \mathrm{C}\right)\end{array}$ \\
\hline $\mathrm{Al}$ & 660 & $0 \sim 150$ & $150 \sim 250$ & $350 \sim 420$ \\
\hline
\end{tabular}

where $a$ is the wave propagation distance and $f$ is a frequency independent function. In addition, in experimental work, the ratio $\frac{A_{2}}{A_{1}^{2}}$, defined as the relative acoustic nonlinear parameter $\beta^{\prime}$, is considered as a measure of acoustic nonlinearity, where $A_{1}$ and $A_{2}$ are the measured amplitudes of the fundamental and second harmonic waves.

$$
\beta^{\prime}=\frac{A_{2}}{A_{1}} \propto \beta a,
$$

The relative acoustic nonlinear parameter increase linearly as a function of propagation distance.

The cumulative effect of the second harmonic amplitude is a great advantage for detection in experimental work. It is found that second harmonic amplitude linearly grows with propagation direction, when synchronism and nonzero power flux. If the wave mode chosen satisfies these two conditions, the second harmonic amplitude will be cumulative. Even a series of double frequency wave components will be generated by the driving sources of nonlinearity. In practice, interest is focused on the second harmonic generation with the cumulative effect, since the cumulative second harmonic wave play a dominant role after second harmonics propagating some distance.

The wave attenuation is defined by an attenuation coefficient. In this work, both the attenuation factors of fundamental wave and second harmonic were tested. The value of attenuation coefficients were used to modify the amplitude of fundamental and second harmonic wave amplitude inversely, thus, this method can minimize the influence of material attenuation on relative nonlinear parameters.

\section{Specimen and Experimental Setup}

\subsection{Experimental Specimen}

The experiment specimen is the Al-6063 pipe, a complex material with outer diameter $20 \mathrm{~mm}$, inner diameter $14 \mathrm{~mm}$, length $323 \mathrm{~mm}$, and thickness $3 \mathrm{~mm}$ (Fig. 2.). Table 1 shows the Aluminum melting point, recrystallization annealing temperature. As shown in Fig. 2, the specimen was loaded for one hour each at $15^{\circ} \mathrm{C}$ $0^{\circ} \mathrm{C}, 250^{\circ} \mathrm{C}, 350^{\circ} \mathrm{C}$.

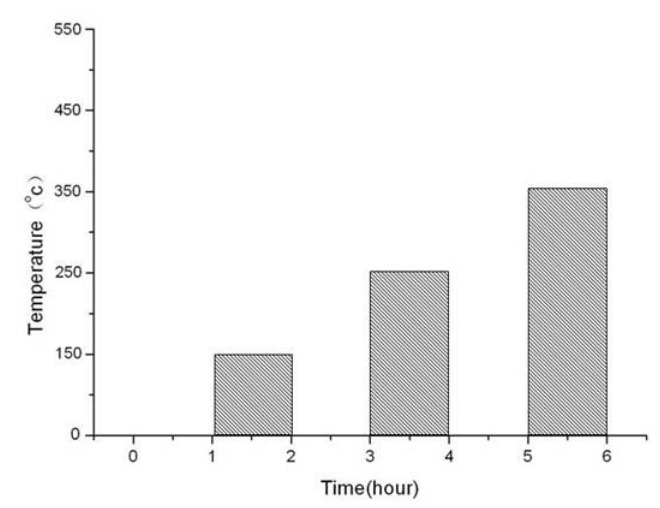

Fig. 2 Heat loading time and temperature

\subsection{Experimental Setup}

Fig. 3 shows a schematic of the experimental setup. The source signal for guided wave generation is a high power function generator (RITEC advanced measurement system RPR4000). The generated waveform is a sinusoidal tone burst with 7 cycles at a central frequency of $1.68 \mathrm{MHz}$, The input frequency is tuned at $1.68 \mathrm{MHz}$, while the central frequency of 
receiver is $3.36 \mathrm{MHz}$ to obtain the corresponding second harmonic frequency components. Flexible polyvinyl-done fluoride (PVDF) comb transducers, which have specific-width electro fingers to control the generated wave length, are coupled to the pipe specimen using an ultrasonic couplant. Fixed pressure is applied on the PVDF transducers to make the couplant thickness consistent.

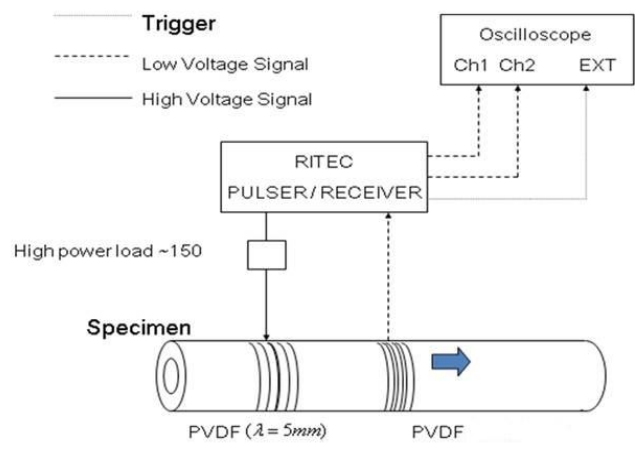

Fig. 3 Schematic of experimental setup
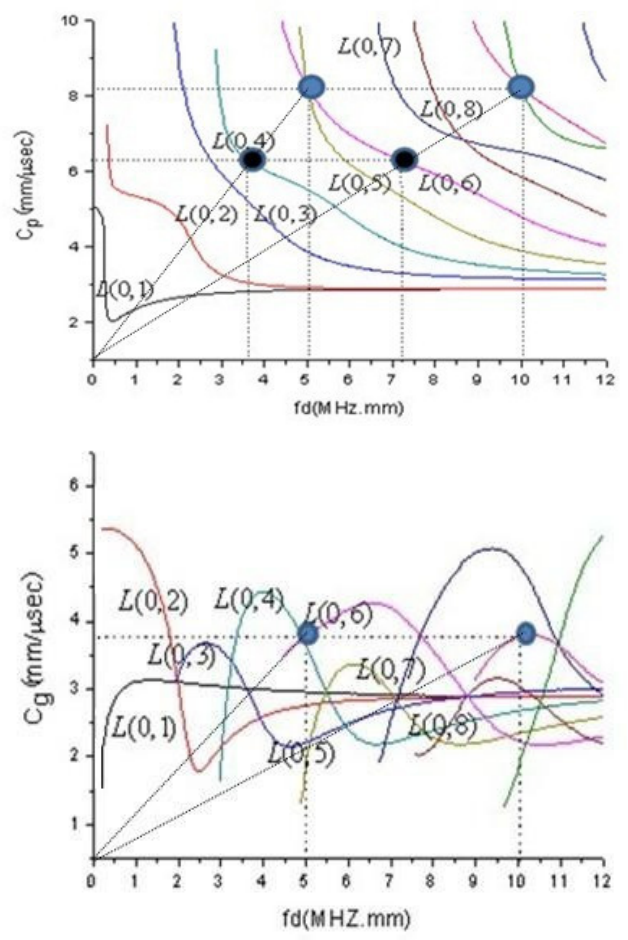

Fig. 4 Numerical dispersion curve of longitudinal mode in the specimen
In dispersion curves Fig. 4, the mode pair $(\mathrm{L}(0,6), \mathrm{L}(0,10))$ is selected in this work. The mode pair satisfies the phase matching condition; the primary wave mode has the same wave velocity as the secondary mode. The mode $\operatorname{pair}(\mathrm{L}(0,6), \mathrm{L}(0,10))$ at $\mathrm{fd}=5.05 \mathrm{MHz} \cdot \mathrm{mm}, \mathrm{fd}=$ $10.1 \mathrm{MHz} \cdot \mathrm{mm}$ with the equal phase velocity of $8.2 \mathrm{~km} / \mathrm{s}$. Therefor the generated fundamental frequency is $1.68 \mathrm{MHz}$, and wave length is $5 \mathrm{~mm}$.

\section{Result and Discussion}

\subsection{Microstructure}

Heat treatment can change the physical and mechanical properties of a material when a material is heated to reply, recrystallization, annealing temperatures. Pictures of microstructural changes of the specimen were obtained by optical microscope as shown in Fig. 5. Variations have been found in a specimen under different heat loading temperatures. However, the microstructural pictures are insufficient to determine out which heat treated specimen is worse. Variations of linear and nonlinear ultrasonic features are tested in different specimens as shown in Fig. 6 and 7. Both the linear and nonlinear acoustic parameters are increase,
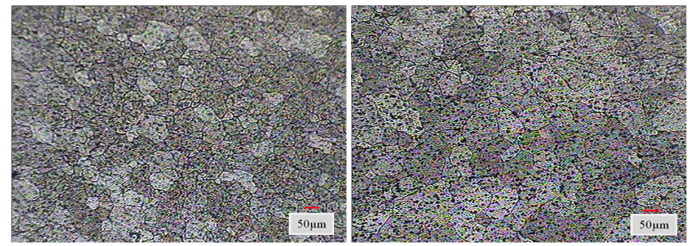

(a) Without heat loading (b) With heat loading $150^{\circ} \mathrm{C}$

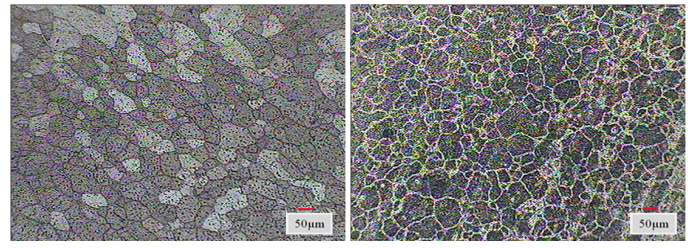

(c) With heat loading $250{ }^{\circ} \mathrm{C}$ (d) With heat loading $350{ }^{\circ} \mathrm{C}$

Fig. 5 Micro-structural pictures of specimens under different heat treatments 


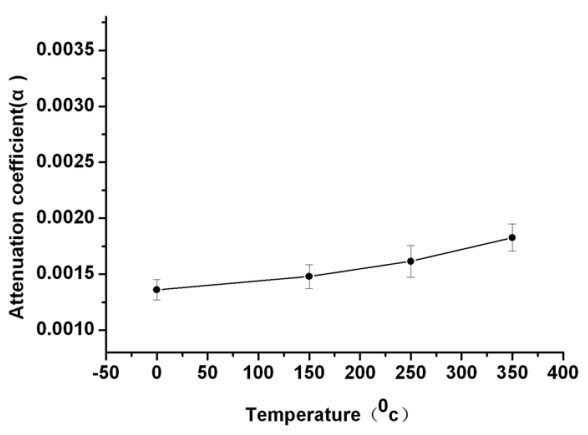

Fig. 6 Linear acoustic parameters of specimen with variation of temperature

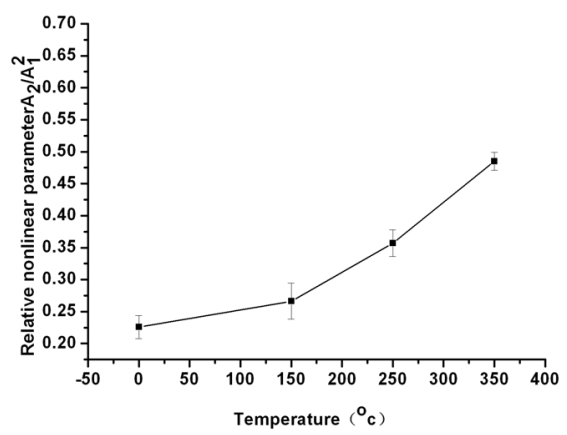

Fig. 7 Nonlinear acoustic parameters of specimen with variation of temperature

however, the comparison of linear and nonlinear parameters in specimens with different heat loading temperatures shows that the variations of nonlinear parameters are more obvious. During the heating process, it shows that grain size decrease, the decrease of grain size can cause the increase of grain boundaries. As known, grain boundary is one of nonlinear source, that is why the material nonlinearity increase. This is the main reason why material nonlinearity increase in this study.

It is important to note that PVDF transducers is a quite nonlinear transducers. When we tested the nonlinear parameters, it definitely includes other nonlinear scources from transducer, equipments. However, In this study, since the guided wave can propagate long distance, as propagation distance increasing, the relateive nonlinear parameters all increasing. The slope ratio can be quantitative relative change after the various thermal damage. This method can be used to minimize the nonlinear source which are not material nonlinearity.

\section{Conclusions}

This paper proposed an experimental setup to detect the thermal damage in an Al-6063 tube-like structure by using ultrasonic guided waves. The variations of acoustic linear and nonlinear parameters were tested in different heat loadings specimens. The experimental results show that heat loading can change the material microstructure; the degradation of material properties was caused by the heat loading.

\section{Acknowledgement}

This work was supported by the Human Resources Development of the Korea Institute of Energy Technology Evaluation and Planning (KETEP) grant funded by the Korea government Ministry of Knowledge Economy (No. 20114010203080) and National Research Foundation of Korea (NRF) grant funded by the Korea government (MEST) (No. 2011-0020812).

\section{References}

[1] S. Suresh, "Fatigue of Materials," Second Ed. Cambridge University Press, New York, USA (1998).

[2] W. Li, Y. Cho and J. D. Achenbach, "Detection of thermal fatigue in composites by second harmonic Lamb waves," Smart Mater. Struct. Vol. 21, No. 8, pp. 085019 (2012)

[3] W. Li, Y. Cho, J. Lee and J. D. Achenbach, "Assessment of heat treated 
Inconel X-750 alloy by nonlinear ultrasonics," Exper. Mech. pp. 1-7 (2012)

[4] J. L. Rose, "Ultrasonic Waves in Solid Media," Cambridge University Press, New York, USA (1999)

[5] D. N. Alleyne and P. Cawley, "The interaction of lamb wave with defects," IEEE Trans. Ultrason. Ferroelectr. Freq. Control, Vol. 39, No. 3, PP. 81-97 (1992)

[6] Y. Cui and D. H. Zou "Numerical simulation of attenuation and group velocity if guided ultrasonic wave in grouted rock bolts," J. Appl. Geophys. Vol. 59, No. 4, pp. 337-344 (2006)

[7] C. Petculescu, S. Krishnaswamy and J. D. Achenbach, "Group delay measurements using modally selective Lamb wave transducers for detection and sizing of delaminations in composites," Smart Mater.
Struct. Vol. 17, No. 1, pp. 015007 (2008)

[8] K. Y. Jhang, "Application of nonlinear ultrasonic to NDT of material degradation," IEEE Trans. Ultrason. Ferroelectr. Freq. Control, Vol. 47 No. 3 pp. 540-548 (2000)

[9] C. Pruell, J-Y. Kim, J. Qu and L. J. Jacobs, "Evaluation of fatigue damage using nonlinear guided waves," Smart Mater.Struct. Vol. 18: 035003 (2009)

[10] M. Deng, "Cumulative second harmonic generation of Lamb mode propagation in a solid plate," Journal of Applied Physics, Vol. 85, No. 6, pp. 3051-3058 (1999)

[11] W. Li, S. Hyun and Y. Cho, "Characterization of ultrasonic nonlinearity by thermal fatigue", Intel. Precis. Eng. Manuf., Vol. 13, No. 6, pp. 935-940 (2012) 